\title{
Response of a Liquid Bridge to an Acceleration Varying Sinusoidally with Time
}

\author{
I. Martínez, J.M. Peralez and J. Meseguer \\ CIDE-E.T.S.I. Aeronáticos, Universidad Politécnica, E-28040-Madrid
}

\begin{abstract}
The response of a long cylindrical liquid column subjected to an axial microgravity field has been experimentally studied on a TEXUS sounding rocket flight to check with theoretical predictions. The expected response of the liquid bridge was a quasi-static amphora-type deformation of the cylindrical shape. However, the experimental results showed a more complex behaviour. Nevertheless it has been possible to find out the reasons of this discrepancy except for a mysterious $0.5 \%$ uncertainty in the stimuli.
\end{abstract}

\section{Introduction}

This experiment belongs to a series (SL-1, TEXUS-12, SL-D1, TEXUS-29, SLD2) ${ }^{1}$ on the mechanics of long liquid columns [1]. The proposal was submitted (Feb-91) after conflicting results were obtained from a related Spacelab-D1 (Nov85) analysis, aiming to elucidate the precise response to an axial acceleration in a presence of g-jitters before the experiments on Spacelab-D2 (Apr-93), although delays on TEXUS shifted it back to Nov-94.

The nominal configuration is a cylindrical liquid column of radius $R(z)=R_{0}$ and length $L$, anchored at the sharp edges of two coaxial solid discs (of radii $R_{0}$ ), held in ambient air by the interface tension $\sigma$. This configuration can only be studied in weightlessness. A silicone-oil column of $R_{0}=15 \mathrm{~mm}, \mathrm{~L}=85 \mathrm{~mm}$, $\left(\sigma=0.02 \mathrm{~N} / \mathrm{m}\right.$, density $\left(\rho=920 \mathrm{~kg} / \mathrm{m}^{3}\right.$ and kinematic viscosity $\left(\nu=10^{-5} \mathrm{~m}^{2} / \mathrm{s}\right.$ is used. The shape should be a perfect cylinder at equilibrium if no forces were applied. But the residual microgravity or g-jitters of the carrying platform (SL or TEXUS) is always present, and not very well known, introducing some uncertainty in the comparison with theoretical predictions. Besides, space experiments are so rare that one tries to explore many parameters, bridge stretching,

${ }^{1}$ TEXUS is the acronym of a German-European sounding rocket program providing up to 6 minutes reduced gravity during free parabolic fall of the order of $10^{-4} \mathrm{~g}_{0}$ with $g_{0}$ the gravity acceleration at the earth surface; SL is the acronym for the Spacelab, a laboratory in the cargo bay of the Space Shuttle; D1 and D2 are acronyms for the German spacelab missions 1 and 2, flown in 1985 and 1993 respectively. (Eds. note) 
vibration, rotation, eccentric rotation, and so on without having time to make redundancy tests, small variations around a working set-point of parameters, and so on.

During SL-D1, one such a liquid column was undergoing shape oscillations in an amphora-type axisymmetric deformation, with an eigenfrequency and a damping as predicted by theory, but with a deformed mid-shape (averaged over the eigenperiod) corresponding to a theoretical acceleration of $\left(70 \pm 10 \cdot 10^{-5}\right.$ $\mathrm{m} / \mathrm{s}^{2}(70 \pm 10 \mu \mathrm{g})$ constantly acting along the column axis. This high value could not be attributed to the Shuttle drag $(<1 \mu \mathrm{g})$ and remains unexplained. A similar experiment was carried out in SL-D2, but then, applying the same analysis, only a value of $5 \pm 1 \mu \mathrm{g}$ was found from the averaged deformation of the liquid column.

\section{Scientific Objectives}

The main goal of this experiment was to provide a calibrated microgravity acceleration $(100 \pm 1 \mu \mathrm{g})$, well above the uncontrolled g-jitters of the platform $(<1 \mu \mathrm{g})$, to check the static response of the liquid column acting as an accelerometer (microgravity accelerometry in the $10^{-3} \mathrm{~Hz}-10^{-1} \mathrm{~Hz}$ range is still controversial). As a constant controlled acceleration $\mu \mathrm{g}$-platform is not available, a low varying sinusoidal motion was foreseen.

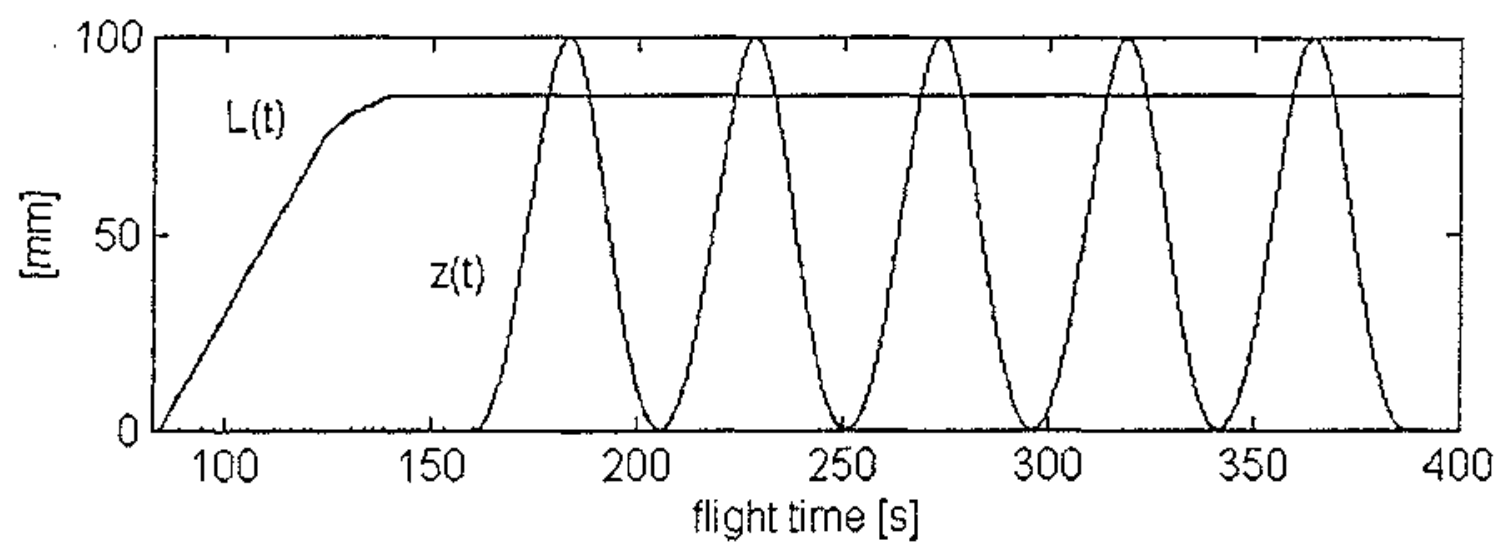

Fig. 1. Disc separation to form the bridge $\mathrm{L}(\mathrm{t})$, and cell oscillations $z(t)$ in the experiment.

The acceleration was imposed by a slow sinusoidal translation of the liquid column cell (already used in previous TEXUS flights) along the common columnrocket axes. From the knowledge of the first natural period of the column (15 s) and optimisation of the microgravity time ( 6 minutes), a forcing period of $T=45$ $s$ was chosen, and a corresponding amplitude of $100 \mathrm{~mm}$ peak-to-peak, in order to reach the acceleration quoted above. Six such cycles were allowed during the 5 minutes time available after the formation of the liquid column in orbit, 
although the first two were expected to be distorted by the initial transients (the half-damping time was known to be some $40 \mathrm{~s}$ ). Lately, the analysis of Spacelab-D2 results prompted us to leave a full 1 minute to study the decay of the oscillations before re-entry and thus the forcing was reduced to 5 cycles, as shown in Fig.1. Observe that oscillations start without velocity jump but with a $100 \mu \mathrm{g}$ acceleration jump. The nominal displacement law was $z(t)=z_{m}(1-\cos (2 \pi t / T))$, with $z_{m}=50 \mathrm{~mm}$ and $T=45 \mathrm{~s}$.

The expected response of the liquid column was a quasi-static amphora-type deformation of the cylindrical shape, best quantified by the radial deformation in a cross-section at $1 / 4$ of the column length $\left(R_{1 / 4}\right)$, as depicted in Fig. 2.

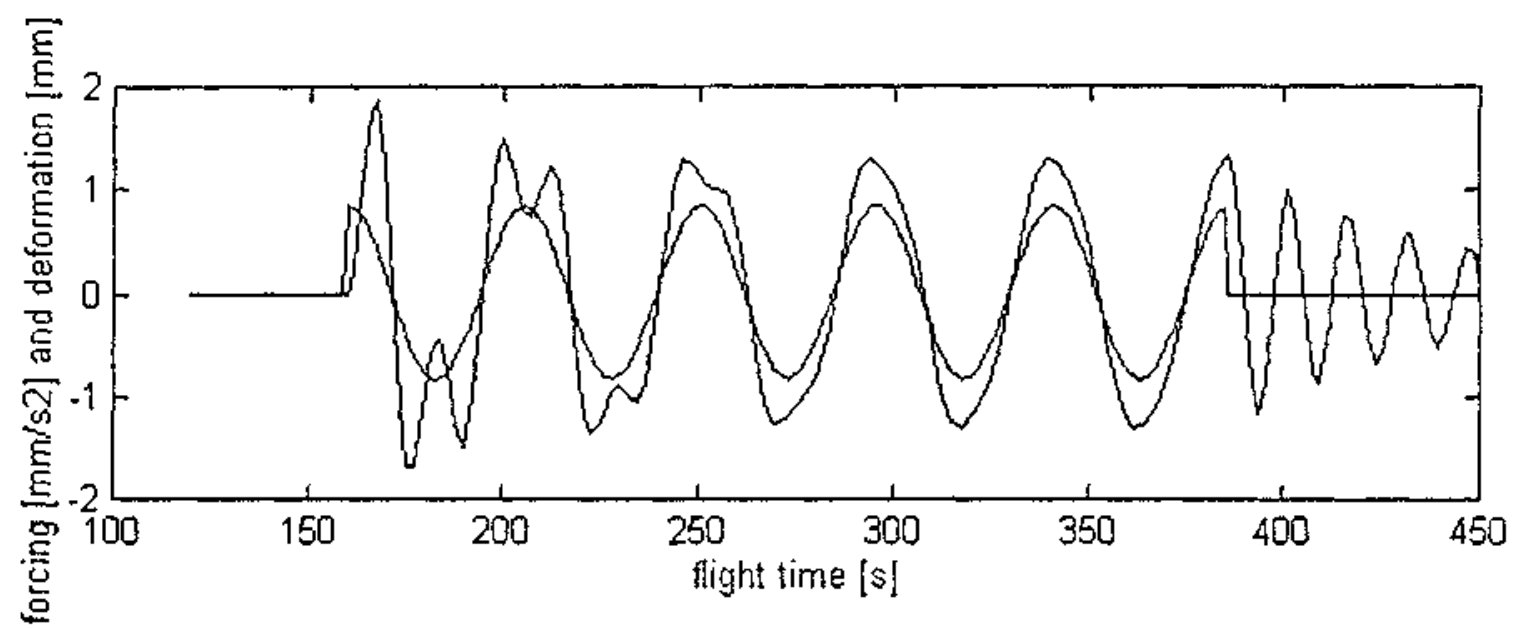

Fig. 2. Ideal excitation (the 5 perfect cycles of the forcing acceleration) and theoretical response of the liquid ( $R_{1 / 4}$ deformation, with damping). After the transients and before the stopping there is a quasi-static ampilication in phase.

Consider an initially cylindrical liquid column subjected to an axial acceleration that we name $\mathrm{g}$ to resemble gravity, although it is orders of magnitude smaller. The equation governing the equilibrium shape is the Young-Laplace non-linear second-order differential equation of capillary pressure (that accommodates the linear pressure profile inside the liquid to the constant value outside):

$$
\sigma\left(\frac{R_{z z}}{1+R_{z}^{23 / 2}}-\frac{1}{R\left(1+R_{z}^{2}\right)^{1 / 2}}\right)=p_{0}+\rho g z
$$

$$
\text { with } \quad R(0)=R(L)=R_{0} \quad \text { and } \quad \pi \int_{0}^{L} R^{2} d z=\pi R_{0}^{2} L
$$

which can be numerically solved [2] for the parameters of this problem $\left(R_{0}, L, \rho, \sigma\right)$ to find first a linear deformation for small $g$ and then a limiting value of $g$ beyond which the bridge breaks ${ }^{2}$. The pressure jump at the origin, $p_{0}$, is an

$\overline{{ }^{2} R_{z}=d R / d z} ; R_{z z}=d^{2} R / d R^{2}$.(Eds. note) 
internal constant to be found from the three boundary conditions of the second order problem. In terms of the Bond number, $B o$, numerical solving (1) yieids $R_{1 / 4} / R_{0}=1+0.009 \cdot B o$ and $R_{1 / 4} / R_{\text {olimit }}=1.19$ (for $B o=B 0_{\text {limit }}=0.018$ ). The linearised analytical solution is:

$$
\frac{R_{1 / 4}}{R_{0}}=1+\frac{L}{4 R_{0}}\left(1-\frac{1}{\cos \frac{L}{4 R_{0}}}\right) B_{0}=1+\frac{\rho R_{0}^{2} L}{4 \sigma}\left(1-\frac{1}{\cos \frac{L}{4 R_{0}}}\right) g
$$

with the definition of the Bond number as $B o=\rho g R_{0}^{2} / \sigma$. This experiment was not the first in the series. A good deal of work was spent not only analysing the nominal configuration but all conceivable possible departures: the influence of an error in the liquid volume injected (from the nominal cylinder), changes in liquid properties, residual spin of the TEXUS rocket $(-3 \mathrm{rpm}$, and a value of $13 \mathrm{rpm}$ would break the column), change in anchoring radii (in case of bad wetting), etc. Even ground simulation by mounting a Plateau tank in a centrifuge was considered, although abandoned for practical difficulties. All the lessons learnt from past experiments were also applied (redundancy of image recording, background grid to aid in image analysis, all information in the image, etc.).

\section{Results}

The results obtained were at first sight all right (large amphora-type deformations), but the detailed analysis was striking, as presented in Fig. 3, due to the fact that the sinusoidal motion was actually achieved by a crank-shaft mechanism, what was not realised by the investigators at the ground tests, where only pen-recorder plots were available (the data acquisition system was not operative).

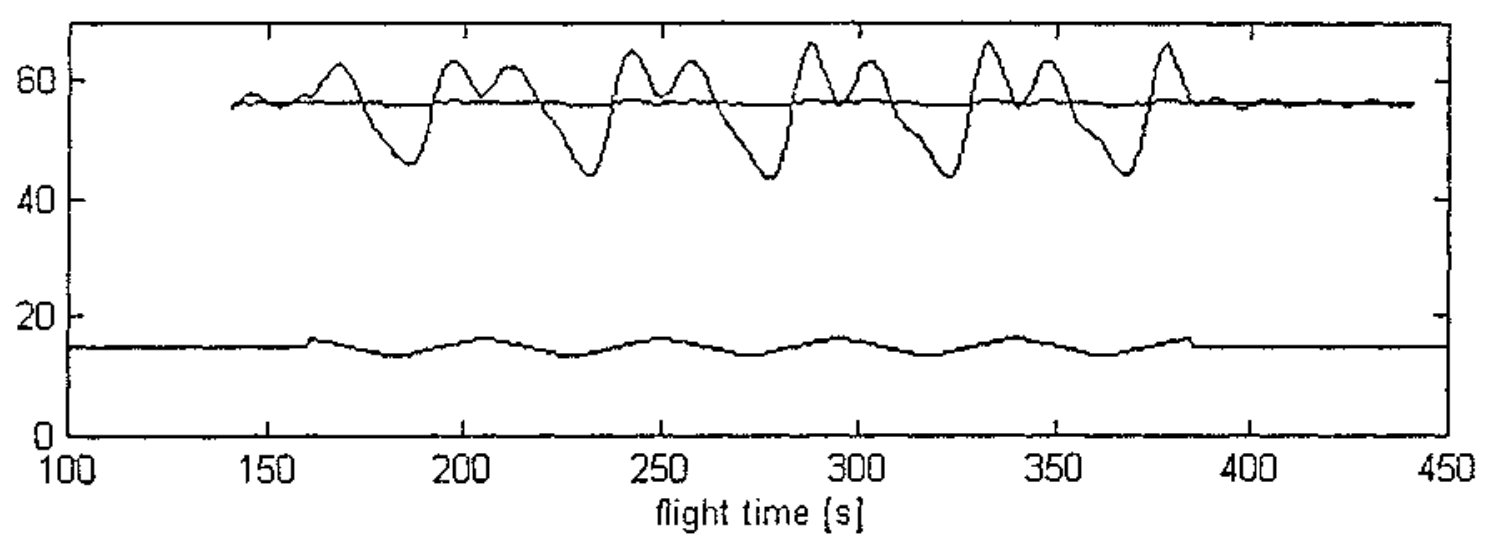

Fig. 3. Discrepancy found in the first analysis of video images. The lower part is the ideal expected $R_{1 / 4}$ deformation in $\mathrm{mm}$, and the upper part the real $R_{1 / 4}$ deformation in pixels. 
The analysis of this experiment is based on the recording of cell displacement by a linear potentiometer and the video recording of images of the liquid bridge against a background reference mask and a fixed reference bar to visualise cell displacement. The potentiometer data (used to draw Fig. 1) has a sampling rate of $0.22 \mathrm{~s}(4.6 \mathrm{~Hz})$ and a resolution of $0.02 \mathrm{~mm}$ in $100 \mathrm{~mm}$ (12 bit), and from that one infers that the actual column length was $\mathrm{L}=85.15 \pm 0.05 \mathrm{~mm}$ (nominally $85 \mathrm{~mm}$ ), oscillations started at $160.4 \pm 0.1 \mathrm{~s}$ flight-time (nominally $160 \mathrm{~s}$ ) and stopped at $386.6 \pm 0.1 \mathrm{~s}$ (nominally $385 \mathrm{~s}$ ), giving a period of $45.2 \pm 0.1$ $\mathrm{s}$ (nominally $45 \mathrm{~s}$ ), with an amplitude peak-to-peak of $99.8 \pm 0.1 \mathrm{~mm}$ (nominally $100 \mathrm{~mm}$ ). The frequency spectrum for the five cycle oscillation is shown in Fig.4, from two redundant sources, as well as that corresponding to the liquid deformation.
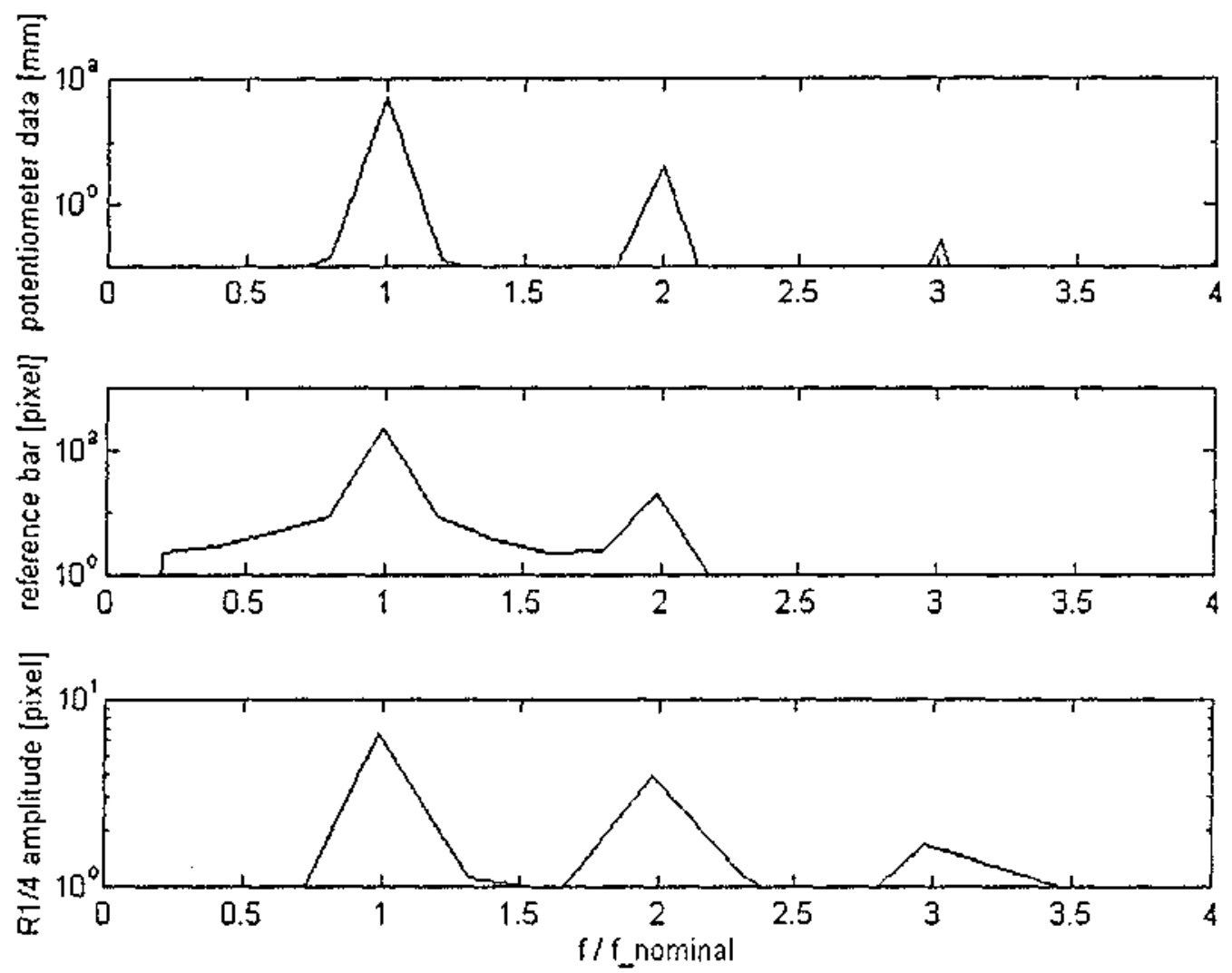

Fig. 4. Fourier analysis of the cell displacement measurement taken by the potentiometer transducer (top), by the reference bar in the image (mid) and of liquid response (deformation at $R_{1 / 4}$ ) (bottom).

Taking into account the uncertainty in the potentiometer data one should disregard the fourth harmonic contribution, but the third harmonic remains, with an amplitude of $0.2 \pm 0.1 \mathrm{~mm}$ still unexplained. From the ratio of the second to the first harmonic, one may deduce the crank shaft length, what yields $158 \pm 10 \mathrm{~mm}$ ( $150 \mathrm{~mm}$ according to design).

The analysis of the video images is not as straightforward as for the potentiometer data, mainly because of the distortions the analogue video signal 
suffers before digitization. For instance, for this experiment, an ideal quiescent cylindrical column once processed (CCD, videotape record, videotape playback, digitization) would present a noisy changing shape with uncertainties of 1 pixel in space and 0.5 pixels in time, although if one only considers odd or even videolines (but not both) and liquid column size (i.e. difference in position of the right and left liquid contours) the uncertainty can be reduced to \pm 0.2 pixels in the image field, corresponding to $\pm 0.05 \mathrm{~mm}$ in the object field.

The video recording is classified according to the interest for further analysis as follows:

- The $53 \mathrm{~s}$ of column formation by liquid injection and disc separation (a small amphora-type deformation is visible, due to the jet injection dynamics).

- The $20 \mathrm{~s}$ of calm-down after filling. It turned out to be too short for a decay of the injection perturbations (the half damping time appears to be $t_{1 / 2} \approx 35 \pm 5 \mathrm{~s}$ ).

- The $90 \mathrm{~s}$ of the two first cycles, where transients from the start-up are distorting the periodic response.

- The 135 s of the last three cycles, practically periodic. Fig. 5 shows a sequence of frames.

- The $60 \mathrm{~s}$ of calm-down after stopping until re-entry. Astonishingly, the deformation after stopping the oscillation is found to be negligible, in contrast to the wide waving expected A perfect cylinder is seen by the naked eye. No residual acceleration is distinguishable in the finest analysis. Natural period appears to be $13 \pm 1 \mathrm{~s}$ instead of the expected $15 \mathrm{~s}$, and half-damping time $\approx 35 \pm 5 \mathrm{~s}$ instead of $40 \mathrm{~s}$ expected.

The axisymmetry, judged by the position and evolution of the centre line (midway between liquid edges at every videoline) is perfect from the beginning up to re-entry of the rocket, where a $\mathrm{C}$-mode deformations grows until breaking the bridge. The antisymmetry of the liquid shape to the mid-plane between the solid discs is also perfect after the long column is established.

Spectral analysis of the fix-reference bar motion digitised from the video images also shows the second harmonic (and a corresponding crank shaft length of $145 \pm 5 \mathrm{~mm}$, but not the third one because a resolution better than one pixel would be needed). The phase of the crank-shaft mechanism, with the flatten humps in acceleration at the upper part of the cell motion (at start-up and stopdown), is responsible for the dead of the oscillations after stopping the stimulus.

The compound oscillations of the whole TEXUS-rocket, the TEM-06-9 module ${ }^{3}$ (fixed to the former by soft mounts), the moving fluid cell and the liquid column itself was analysed: $m_{\text {liquid }}=0.06 \mathrm{~kg}, \mathrm{~m}_{\text {cell }}=10 \mathrm{~kg}, \mathrm{~m}_{\text {module }}=60 \mathrm{~kg}$, $m_{\text {rocket }}=400 \mathrm{~kg}$. From the $\mu \mathrm{g}$-accelerometers in the rocket, a train of pulses appear synchronised with our experiment timeline, with pulse amplitude precisely synchronised with the peaks in speed of our cell displacement, and not with acceleration, what might be explained by a series of beating of a loose joint at its bounds.

${ }^{3}$ TEM is the acronym for a TEXUS Experiment Module. The module 06-9 is a special module for fluid physics experiments. (Eds. note) 

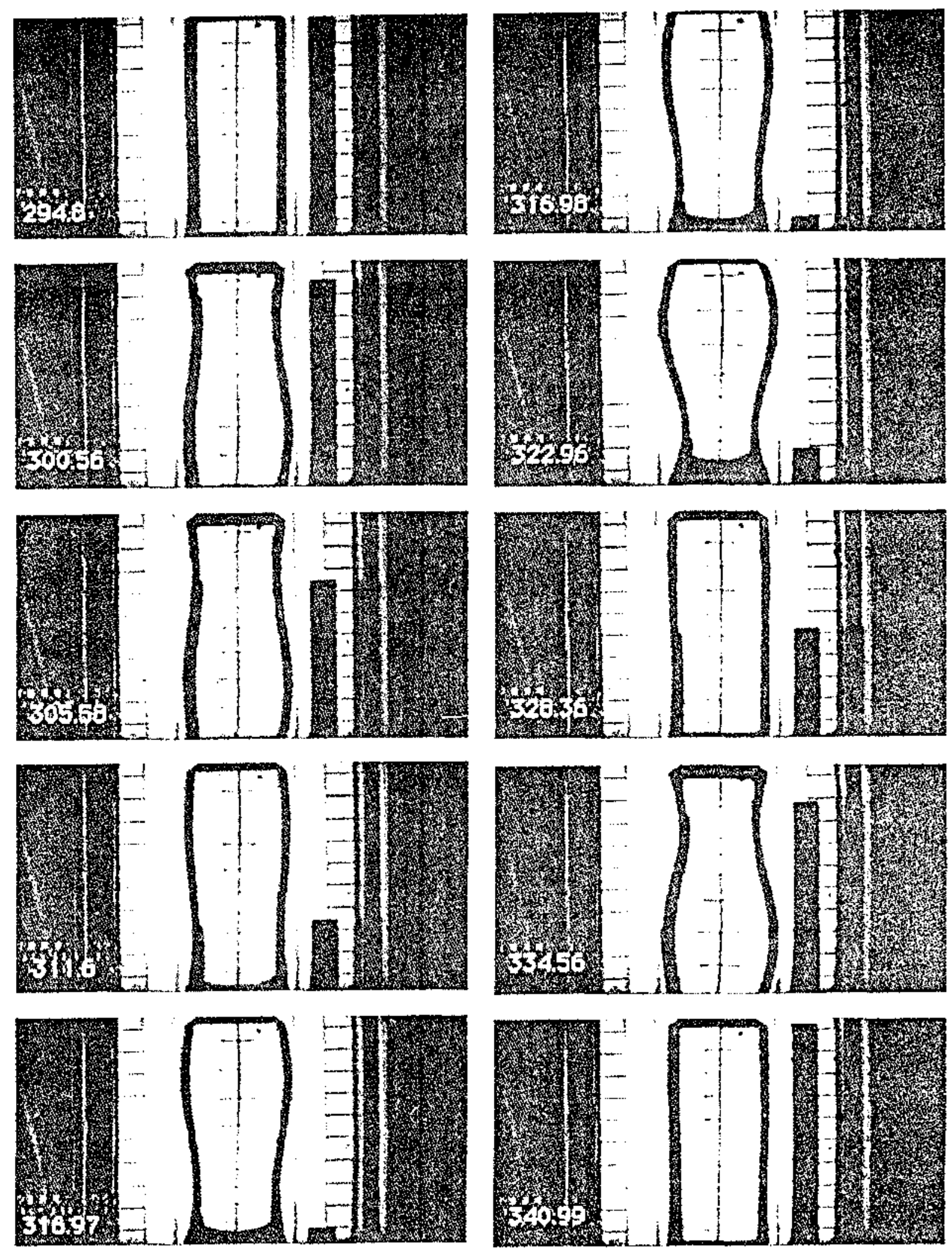

Fig. 5. A complete cycle of $45 \mathrm{~s}$ in the response of the liquid bridge starting at $294.2 \mathrm{~s}$ flight time. 
A one-dimensional dumped-spring model is established to simulate the linear dynamics of the liquid column, once that available liquid bridge theory [3] shows that non-linear effects should be negligible and that the only relevant mode is the first (amphora-type oscillations):

$$
\frac{d^{2} x(t)}{d t^{2}}+\frac{2 \ln 2}{t_{1 / 2}} \frac{d x(t)}{d t}+\left(\frac{2 \pi}{T}\right)^{2} x(t)=K\left(\frac{2 \pi}{T}\right)^{2} \ddot{z}(t)
$$

where $x$ is the $R_{1 / 4}$ deformation $\left(R_{1 / 4}-R_{0}\right)$, 2 the cell displacement, $t_{1 / 2}=40$ $\mathrm{s}, T=15.3 \mathrm{~s}$ and $K=0.24$ deduced from (1) for the static case. The gain in the frequency response of this linear system is shown in Fig.6.

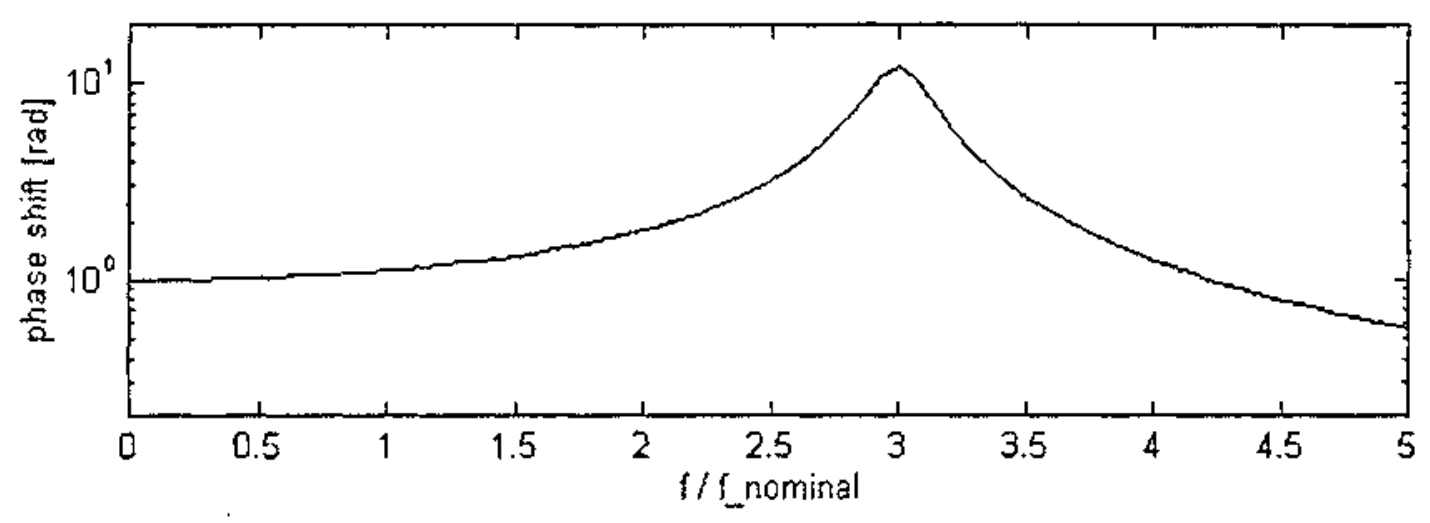

Fig. 6. Frequency response in amplitude of liquid deformation relative to amplitude of stimulus ( $f_{\text {nominal }}$ corresponds to the $T=45 \mathrm{~s}$ cycling at a third of the eigenfrequency).

Inclusion of the second harmonic from the crank-shaft mechanism explains the camel-shape response in Fig.3, but there still remains a small third harmonic contribution (some $0.5 \%$ of the intended displacement amplitude) that is pervasive to the analysis so far performed. The best matching achieved is shown in Fig.7.

\section{Conclusions}

Most aspects of the response of a highly sensitive long liquid column to a controlled acceleration have been understood in great detail with the theory developed so far. Only two puzzling questions remain open:

1. an artificial contribution of $0.5 \%$ in the applied stimuli to the third harmonic (and maybe of $0.4 \%$ to the forth), is found to better match the real liquid response than the mere crank-shaft motion.

2. a phase shift of precisely $\pi / 2$ in the acceleration measured in the rocket body and the one applied. 


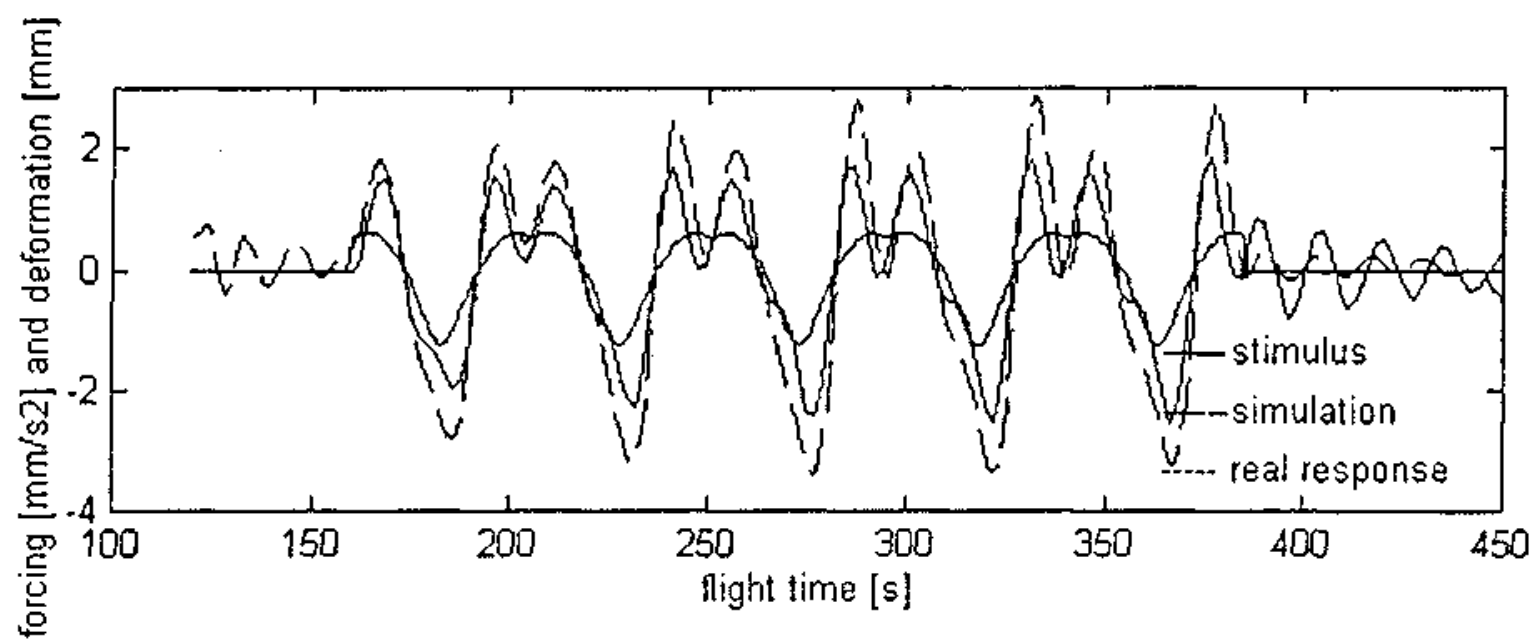

Fig. 7. Real excitation (the humped 5 cycles), real response of the liquid (from video) and expected response (from numerical simulation with (3) and third and forth harmonics).

Some lessons have been learnt for future work: 1) even at the high cost of space experiments, time periods to damp-out initial perturbations or to achieve periodic response must be generous, and 2) a negligible $0.5 \%$ noise in relative displacement at the eigenfrequency, when driving the system at one third of it, means a $3^{2} \cdot 0.5=5 \%$ noise in relative acceleration due to the centrifugal effect, and selectively amplified 12 times due to the weak damping, finally yields a $12 \cdot 3^{2} \cdot 0.5=50 \%$ noise in relative response of the liquid bridge.

\section{Acknowledgement}

The authors wish to acknowledge the invaluable help of the students E. Moreno, J. Paino and M. Ramos, the ESA and ERNO TEXUS teams, and the financial support of the Spanish Grant CICYT ESP92-0001-CP.

\section{References}

1. I.Martinez, J.M. Perales and J. Meseguer, Stability of long liquid columns, in: Scientific Results of the German Spacelab Mission D2, P.R. Sahm, M.H. Keller, B.Schiewe (Eds.), DLR, Köln, Germany 1995, p.220

2. J.M. Perales, J. Meseguer and 1. Martinez, Minimum Volume of Axisymmetric Liqujd Bridges between Unequal Disks in an Axial Microgravity Field, J. Cryst.l Growth, I10 (1991) 855-861.

3. J. Meseguer and J.M.Perales, Non-steady Phenomena in the Vibration of Viscous Cylindrical Long Liquid Bridges, Microgravity Science and Technology, 5 (1992) 69-72. 